\title{
Talking over a beam of light: electro- optics suitable for K-12
}

\section{Michael Nofziger}

Michael J. Nofziger, "Talking over a beam of light: electro-optics suitable for K-12," Proc. SPIE 2525, 1995 International Conference on Education in Optics, (13 October 1995); doi: 10.1117/12.224016

SPIE Event: SPIE's 1995 International Symposium on Optical Science, Engineering, and Instrumentation, 1995, San Diego, CA, United States 


\title{
"Talking over a beam of light!": electro-optics suitable for K-12
}

\author{
Michael Nofziger \\ Optical Sciences Center \\ University of Arizona \\ Tucson, Arizona 85721 \\ Electronic Mail: nofziger@gas.uug.arizona.edu
}

\begin{abstract}
A kit of electro-optical components is described which, when assembled and aligned, allows one to transmit voice signals over a beam of light. The kits were developed for TOPTIC ' 92 , an optics convention held in the city of Tucson to showcase local optical companies and education opportunities to the public-at-large. One aspect of the convention was to involve school children with the world of optics. These kits proved to be an excellent way to link educators and their students with parents, optics professionals, and even the local media.

The kits consist of all the necessary electrical components to build a transmitter (using an LED) and a receiver (using a phototransistor). The circuits are assembled on an electronic breadboard which is part of the storage case for the parts themselves. Optically, the light beam is collimated and focused using inexpensive Fresnel lenses. Distances over which one's voice may be sent are variable, determined by one's ability in optical alignment. Students in the 5 th grade were the first to use the kits, sending their voices about 200 feet. The educational success of these kits is described in this paper.
\end{abstract}

Keywords: optics education, communication, kit, K-12

\section{BACKGROUND}

In the fall of 1992, the Arizona Optics Industry Association (AOIA) teamed up with the city of Tucson to host the "Tucson Optics Convention," called TOPTIC '92. One feature of the two-day event was that of optics in education. The goal of AOIA was to introduce some aspect of optics at the middle school level, providing students with a hands-on experience. The final evening of TOPTIC ' 92 was to include a forum where the students could showcase their experiences to their parents, optics professionals, and the public at-large. This author had taught optics to middle school students as part of a local summer program, ${ }^{1}$ and was contacted by AOIA to help with TOPTIC '92.

The exact nature of the educational efforts first needed to be defined. Time was a factor, as the convention was to take place in a couple of months. It was decided at the outset that we would focus on one science class at a local middle school, involving approximately 35 students and their teacher. Various initial ideas were considered, including letting each student work on a topic of their choice, concluding with a "mini" science fair. This idea and others were rejected based on the limited time to the convention, and perhaps more importantly, the time that a given teacher would want to dedicate to one particular subject such as optics. The goal was to be supportive of the teacher's efforts, and not burden their existing curriculum. 
After much thinking, this author decided to develop an electro-optics kit suitable for the 5th graders to be involved, yet flexible enough to be used later on for students in K-12. The kit would contain all parts necessary to build a circuit that allows one to send his/her voice over a beam of light. The circuit is one published in a book by Radio Shack entitled "Engineer's Mini-Notebook: Optoelectronics Circuits," written by Forrest M. Mims III. ${ }^{2}$ The circuit was breadboarded, tested, and found to work as advertised. Not only that, but it was fun to build and use! Funding for the parts was to be provided by AOIA, within some reasonable budget. The total cost for 10 complete kits came to $\$ 500$.

We met with the entire science department at the middle school, making a point to invite all of the teachers (not just those who might be interested in electronics or optics). After introducing the idea that we wanted to provide a kit to help teach a unit on optics, much interested discussion ensued. The teachers immediately wanted to know if we had a teacher's guide developed, perhaps a video instruction. Of course the answer was "no" as in fact the kits at this point in time were still an idea waiting to happen! Some of the teachers pointed out their lack of knowledge about electronics, others to the lack of a teacher's guide, others were reluctant to work on such short time notice, but in the end, one teacher expressed her desire to help. She was the type of person who wasn't afraid to try something new on short notice, which fit our efforts perfectly!

The kits were developed over the next couple of weeks. To keep costs as low as possible, parts were identified and purchased from the surplus markets. To someone not familiar with surplus buying, the term usually conjures up images of used, dirty, broken parts, i.e. "junk." In fact, many if not most surplus items are new items being sold as overstock or outdated for some particular need. For purposes of putting together these kits, surplus buying was a wonderful way to keep the costs down. (It is true that this probably wouldn't be appropriate to develop a kit such as this on a large-scale, because often surplus is of limited quantity--when it's gone, it's gone.) Some of the parts were purchased locally, others via mail-order, and a few had to be bought new (batteries, for example!).

While this was taking place, this author went to the class on five separate occasions to teach mini-units on basic electronics and optics. The concepts of voltage, current, resistance, and Ohm's Law were covered, as well as the functions of resistors, capacitors, transistors, and the integrated circuits contained in the kits. Basic optics of lenses were introduced, including the concept of focal length of a positive lens. Fresnel lenses were talked about, as these were to be used in the kits. It became immediately obvious that the students were motivated, highly interested, and eager to learn about optics! They wanted to start on the kits as soon as they were ready!

\section{THE KITS}

This section contains details of the receiver and transmitter kits. Keep in mind that the actual implementation of the circuits could vary, depending on the method of construction and available funds. In these kits, electronic breadboards are used because of their relative low cost for added versatility. For those not familiar with breadboards, think of them as a matrix of connected holes, wired together as columns. These particular breadboards have a single row of connected holes along the top, and a similar row along the bottom. The center of the board has a solid area, which divides the upper set of columns from the lower set. In this way, each pin of an integrated circuit (placed so as to straddle this solid area) makes connection with a unique column. Four other parts may then be connected to each pin.

These breadboards were modified for use with the receiver and transmitter circuits. The top row of holes was 
broken into two halves, and wired directly to two $9 \mathrm{~V}$ batteries through an ON/OFF switch. The left half connects to $-9 \mathrm{~V}$, the right half to $+9 \mathrm{~V}$, to power bipolar op-amps. The bottom row was left intact as a connection to ground. Further, a few of the columns at the right edge of the board make specific connections to the input and output jacks, as well as the potentiometers. While reducing the versatility of the breadboards somewhat, this greatly simplifies the wiring. Perhaps the greatest expense of doing this is that the connections to these parts become "hidden" to the student. Even so, the purpose for making each connection can be described in the step-by-step manual.

Once the breadboard, storage box, and batteries are purchased (the major expenditures), a variety of electrooptical circuits can be built for just the additional cost of electronic parts (very cheap these days). (Plans are underway to develop an entire lab of easy-to-build opto-electronic circuits for grades K-12.) ${ }^{3}$

Keep in mind that each transmitter and receiver kit was designed to be built by two students. With 10 complete kits available, this accomodates a total of 40 students, greater than the typical middle school class size.

\subsection{The Receiver}

The schematic drawing of the receiver, modified slightly from the book by Mims, is shown in Figure 1. A picture of the receiver, as built by the students, is shown in Figure 2. The receiver is assembled step-by-step, beginning with the two integrated circuits. Resistors and capacitors are added next, followed by the interconnecting wires (the wires are extra long to make connections easier). This has been found to be easier than to have to add parts under wires already in place. In fact, the greatest difficulty students have in fixing problems found during troubleshooting is trying to reposition parts underneath the wires.

Essentially, the receiver consists of two op-amps. The first functions as a transimpedance amplifier to convert the detector current into a voltage. The second is an audio amplifier, providing enough gain to drive a speaker typically used as a mini desk-top speaker for a Walkman-type personal stereo (these speakers were bought on the surplus market as a stereo pair, and the cables were then cut in half and re-wired to give "two speakers for the price of one." The price for each of these speakers in a nice plastic cabinet was lower than the cost for just one speaker of equivalent size.)

Experience has shown that most students are able to build the receiver correctly on their first try. Still, each unit should be "inspected" before handing out the phototransistors and speakers. The easiest way to troubleshoot the circuit is to have a pre-built working version in-hand, and simply compare the two. This is much easier than trying to read parts connections from the schematic, something which becomes immensely important when trying to troubleshoot 10 circuits practically at the same time! It might be noted that the most common error seems to be installing the integrated circuits in the wrong holes, or even backwards. Another typical error is to install the one electrolytic capacitor backwards. Checking this early in the construction process will save problems later on.

Once the unit has been checked, the phototransistor and speaker may be connected. After turning the unit on, a buzzing sound will be heard if overhead fluorescent lights are on. The sound is, in fact, coming from the 120 $\mathrm{Hz}$ modulation of the emitted light. The more light incident upon the phototransistor, the louder the sound (also, the higher the volume control, the louder the sound). Be prepared for a roomful of speakers all blaring $120 \mathrm{~Hz}$ tones!) The next part provides room for experimentation. Have the students detect various sources 
of light, to see if any make other kinds of sounds. Easy ones to find include tungsten bulbs, the sun, LED's, and TV or computer screens.

A word of warning here: the permanent magnet in the speakers can cause a "permanent" magnetization of the front mask in a large-sized TV or computer monitor, leaving the color balance "permanently" messed up. Keep the speakers away from the screens!

It is interesting to "hear" the differences between light emitted from fluorescent and tungsten bulbs. The fluorescent bulbs emit a strong $120 \mathrm{~Hz}$ modulation, while very little is heard from the tungsten bulbs (as is to be expected). This is a great opportunity to explain the mechanical and optical differences between the two types of bulbs!

\subsection{The transmitter}

The schematic drawing of the transmitter, as taken from the book by Mims, is shown in Figure 3 . A picture of the completed transmitter, built by the students, is shown in Figure 4. The transmitter is built step-by-step, beginning with the one integrated circuit and the transistor. It has become apparent from experience that the transistor is the single most difficult part to install correctly. It, along with proper installation of the integrated circuit, should be checked early in the assembly process. The resistors and capacitors are installed next, followed by the wires. This circuit contains two polarized electrolytic capacitors, which need to be installed correctly and should also be checked by the instructor.

The transmitter consists of an electret microphone feeding an audio amplifer, and a transistor which drives current through the LED to amplitude modulate the emitted light. In effect, this is an AM transmitter operating at the wavelength of the LED! Yellow LED's are used in order to make optical alignment at large distances easier (yellow being near the peak spectral response of the human eye).

Each of the transmitters should be "inspected" before the LED's and microphones are handed out. As with the receivers, the easiest way to troubleshoot the circuit is to have a pre-built unit on-hand for comparison.

\subsection{Testing the link}

Once the students' circuit is inspected, the LED and microphone may be connected and the unit checked for proper operation. The LED should increase and decrease in brightness in step with the amplitude of the voice speaking into the microphone. At this point, the link my be tested "end-to-end" by placing the LED and phototransistor adjacent to each other. Students quickly attempt this, and delight in hearing their voices come from the speaker. The feeling of accomplishment will be written all over their faces! The final step is to use the Fresnel lenses to increase the distance between transmitter and receiver. Students should already have measured the focal length of these lenses, and can place them this distance in front of the LED and the phototransistor, keeping each component on an imaginary optical axis. Note that wooden fixtures were made to hold the LED, phototransistor, and the two Fresnel lenses such that the center of each is kept at the same height. In this manner, the optical alignment is reduced to maintaining a "straight" optical axis. (In practice, some height adjustment always seems to be necessary, and the posts holding the LED and phototransistor allow for up and down movement.) Figure 5 shows the completed link, end-to-end. 


\section{TEST RESULTS}

As with any engineering task, the proof of success ultimately lies with the user's ability to make use of the equipment designed. These kits (and kit design in general) are no exception. Success is measured by how easily the student can use the kits, how quickly the kits can be made to work with no excessive amount of frustration, and how much is learned from the entire process. The 5th grade class taking part in TOPTIC '92 was the ultimate beta test for these newly-put-together kits.

The day arrived when the receiver kits were placed in the hands of each group of two students. Following the instructions, the students went to work almost immediately. Although it was never planned this way, an atmosphere of friendly competition quickly developed to see who could build their kit the fastest. After about 40 minutes, a distribution of results were obtained. There were two groups who had finished, one group who had barely installed two parts, with the other groups falling in-between. The groups who were finished were given the detectors and speakers, and quickly filled the room with the buzzing of fluorescent lights. This had an amazing effect--almost all of the other groups picked up their pace, wanting to experience the same success! This had to wait until the next class period, during which all of the groups finished their receivers. The same scenario was played out while building the transmitters, and after another class period most groups had working links, at least over a short distance!

Another class period was devoted to seeing how far apart the units could be separted and still hear the transmitted voices. The students were allowed to work in the hallways, where they quickly discovered that the floor tiles provided easy alignment marks, even over large distances! It wasn't long until some of the links were working over distances of 50-75 feet. A couple of the more careful students were able to achieve a separation of about 150 feet, in part limited by ambient lighting and also time constraints of the class.

\subsection{The final demonstration}

In effect, the fun had just begun. What was left was for the students was to show off their hard work to the participants of TOPTIC ' 92 , including their parents, optics professionals, and the public-at-large. Banners and posters were made by the students describing their efforts. As it turned out, some of the students who were not as mechanically inclined in building the kits were better at contributing artistic efforts in making the posters.

The forum chosen by the organizers of TOPTIC was that of an evening set aside to showcase optics in education around the Tucson area. Part of the Tucson Convention Center was set aside for this particular evening. Out of convenience, we had our students set up their voice links on the carpeted floor of a large foyer, leading to a hall where a featured speaker was to demonstrate a variety of toys that make use of optics. As luck had it, this was an excellent location! Every person, adults and kids alike, who walked into the lecture hall had to step across a number of beams of light, each carrying the voices of excited students. This got the attention of people in a way that wouldn't have happened if the kits had been set up in some corner of the foyer, out of the way of traffic. Instead, our students had many opportunities to explain what they had set up and to demonstrate just how their links worked. The evening ended for our students in a positive way not thought possible by anyone involved in bringing this project to completion. Local television media covered the event, to include close-up shots of electronic parts, beams of yellow light, and excited looks on the faces of an entire middle school class! 


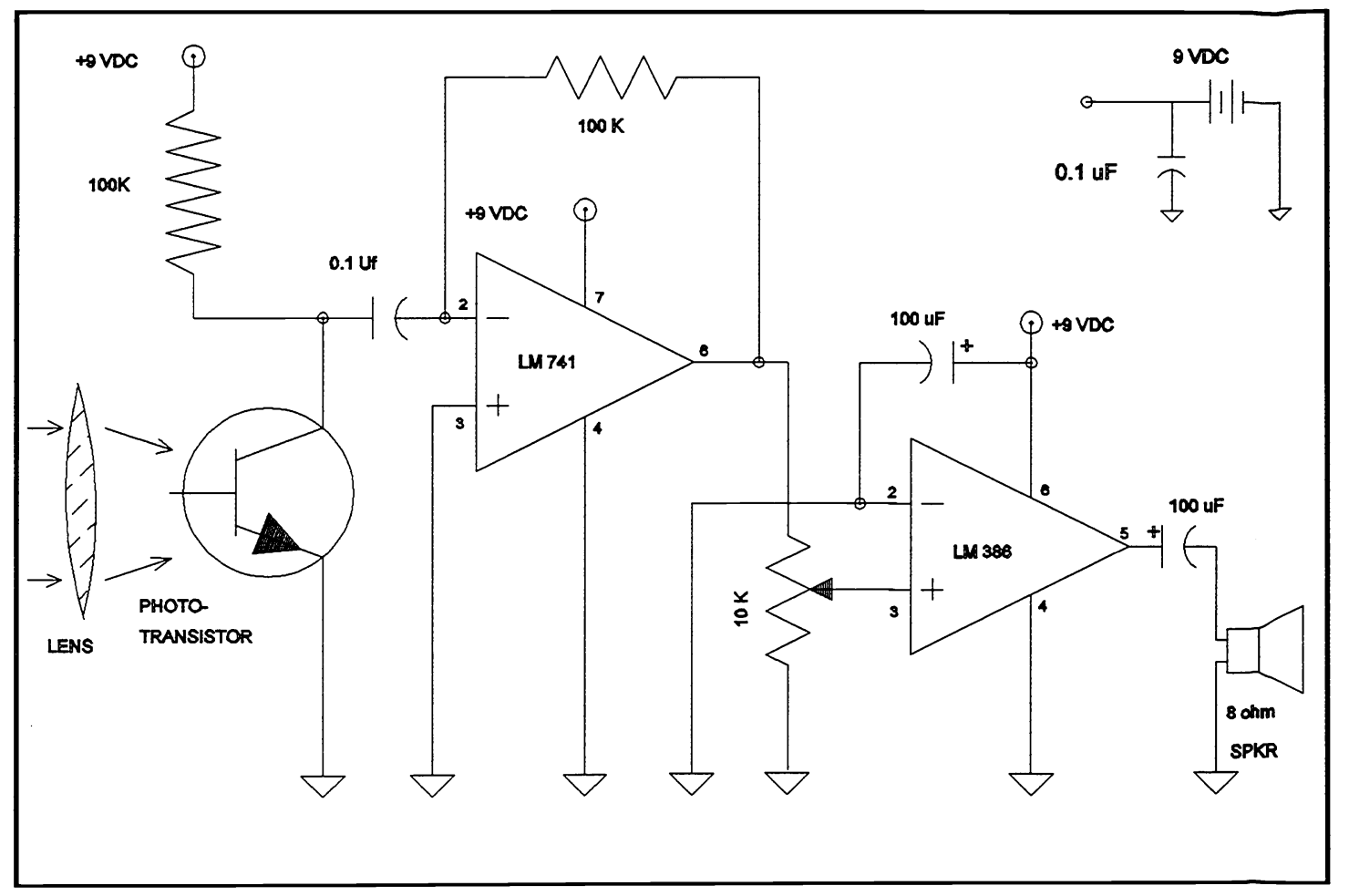

Figure 1. Schematic diagram of the receiver

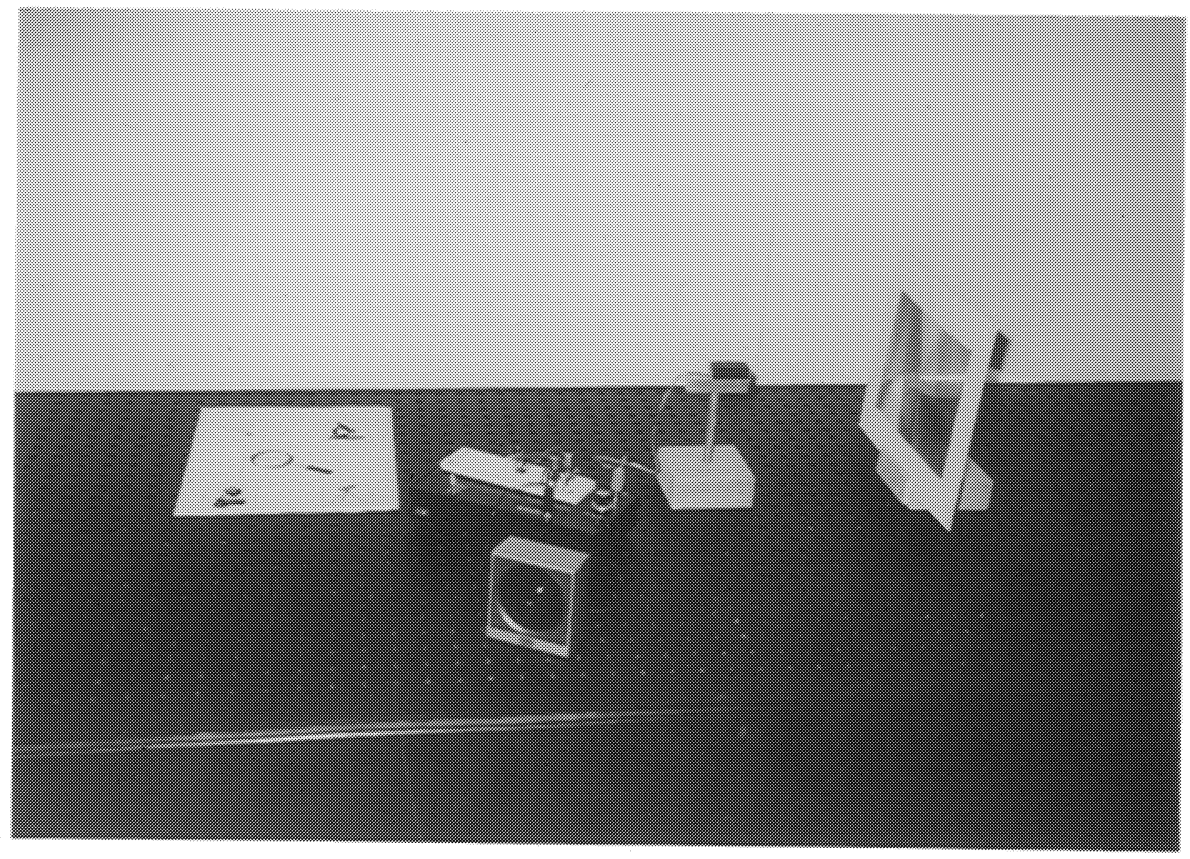

Figure 2. The reciever kit 


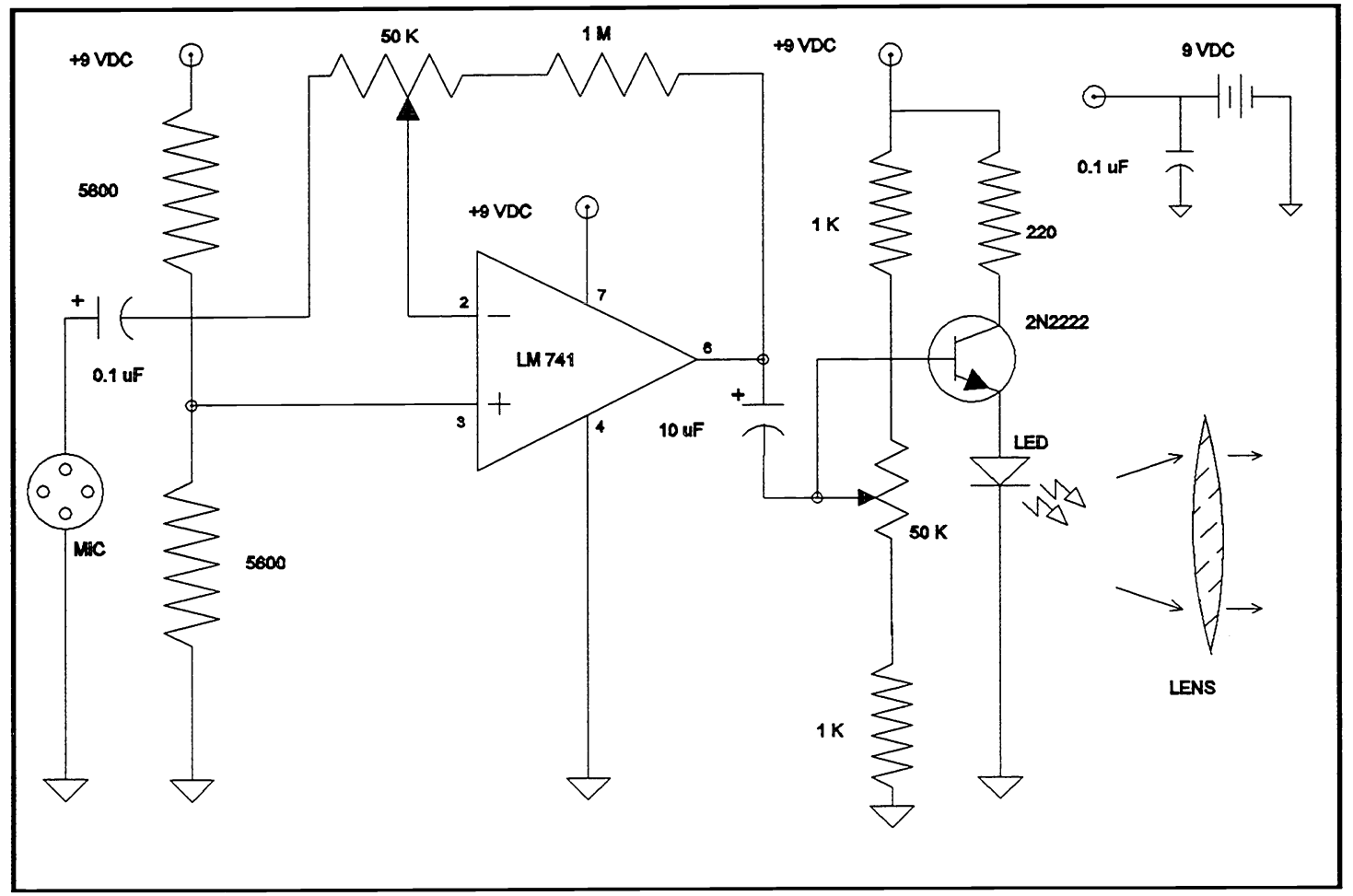

Figure 3. Schematic diagram of the transmitter

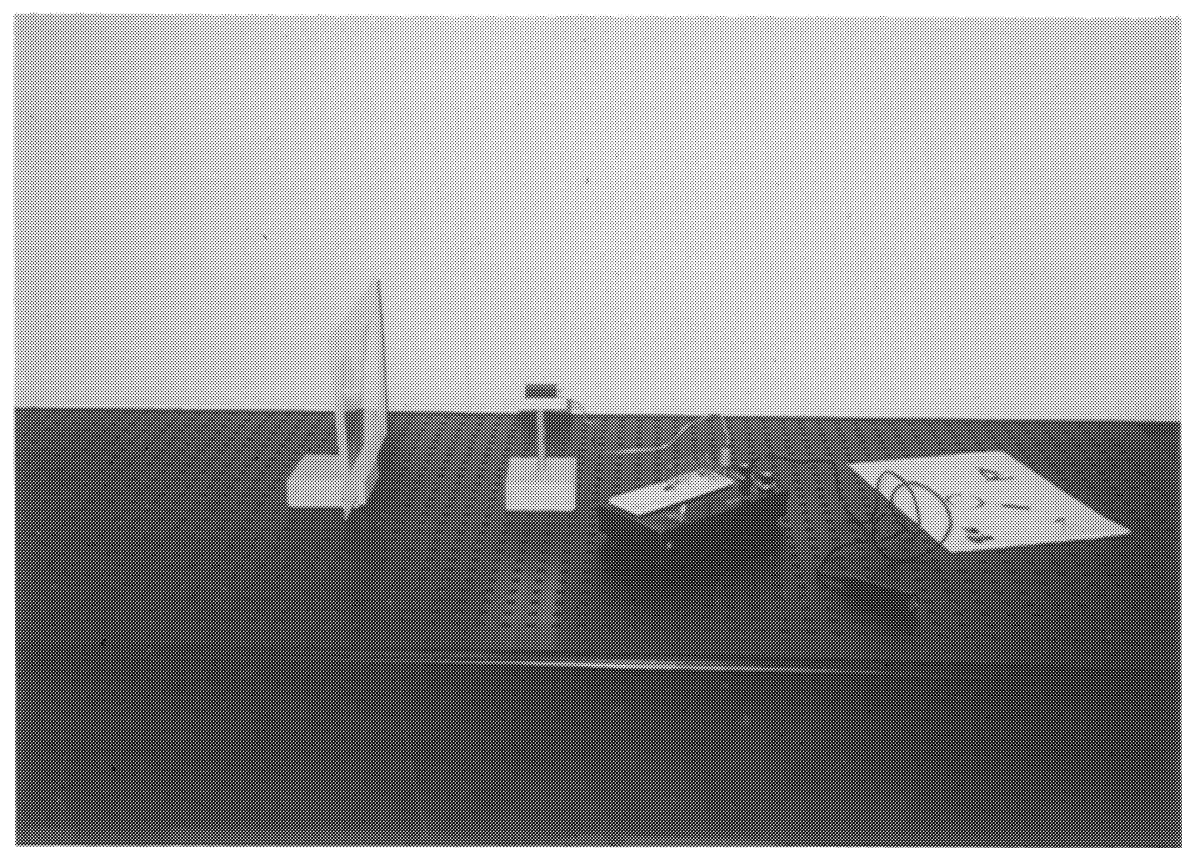

Figure 4. The transmitter kit 


\section{REFERENCES}

1. M.J. Nofziger, "Optics curriculum for middle school students," Proc. SPIE 2525 , paper no. 76 (1995).

2. F.M. Mims III, "Engineer's mini-notebook: Optoelectronic Circuits," Radio Shack catalog no. 276-5012.

3. Contact the author for further details. 